\title{
Properties of the survival implications and S-implications
}

\author{
Piotr Helbin, Michał Baczyński
}

Institute of Mathematics, University of Silesia, 40-007 Katowice, Bankowa 14, Poland

\begin{abstract}
Recently, Grzegorzewski [9] introduced two new families of fuzzy implication functions called survival implications and survival S-implications. These two classes of multivalued implications are based on conditional copulas. In the same article the author gave a motivation to his ideas and he analyzed some properties of these classes of fuzzy implications. In this paper the laws of contraposition and T-conditionality are studied for these families of fuzzy implications. Furthermore, we discuss the intersections of both new families of implications with R-implications and (S,N)-implications.
\end{abstract}

Keywords: Copula; Fuzzy implication; Survival implication; Laws of contraposition; Tconditionality; Functional equations.

\section{Introduction}

Fuzzy implications belong to the main logical operations in fuzzy logic. They generalize the classical implication, which takes values in the set $\{0,1\}$, to the unit interval $[0,1]$. These functions are not only essential for fuzzy logic systems and fuzzy control, but they also play a significant role in solving fuzzy relational equations, in fuzzy mathematical morphology and image processing, and in defining fuzzy subsethood. In the scientific literature (see the monograph [3] and the very recent book [1]) one can find many examples of families of fuzzy implications along with the investigations on their properties and applications.

Recently, Grzegorzewski $[7,8,10]$ introduced two new families of fuzzy implications based on copulas - probabilistic implications and probabilistic Simplications. These families have been further investigated in [2, 9]. Independently, in [9], Grzegorzewski introduced next two families of fuzzy implications based on copulas - survival implications and survival S-implications. He also examined when these functions are fuzzy implications. In this paper we continue the above investigations and we examine the laws of contraposition, principle of T-conditionality and intersection with Rimplications and $(\mathrm{S}, \mathrm{N})$-implications for these two families of functions.

The paper is organized as follows. Section 2 contains some preliminaries, where we recall the basic concepts and definitions used in the paper. In Section 3 we discuss the laws of contraposition for survival implications and survival S-implications, respectively. In Section 4 we examine the principle of T-conditionality for these two families of implications. In Sections 5 we discuss the intersections of survival implications and survival S-implications with R-implications and (S,N)-implications, respectively. Last section contains the conclusion and the postulate some open problems.

\section{Preliminaries}

According to the well-established fact that fuzzy concepts have to generalize adequately the corresponding crisp concepts, the most commonly accepted definitions of fuzzy connectives are the following.

Definition 2.1 ([3, Definition 1.1.1]). A function $I:[0,1]^{2} \rightarrow[0,1]$ is called a fuzzy implication if it satisfies the following conditions

(I1) $I$ is antitone with respect to the first variable,

(I2) $I$ is isotone with respect to the second variable,

(I3) $I(0,0)=I(1,1)=1$ and $I(1,0)=0$.

Definition 2.2 ([11]). A function $T:[0,1]^{2} \rightarrow[0,1]$ is called a triangular norm (t-norm) if it satisfies, for all $x, y, z \in[0,1]$, the following conditions

(T1) $T(x, y)=T(y, x)$,

(T2) $T(x, T(y, z))=T(T(x, y), z)$,

(T3) $T(x, y) \leq T(x, z)$ for $y \leq z$, i.e., $T(x, \cdot)$ is isotone,

(T4) $T(x, 1)=x$.

Definition 2.3 ([11]). A function $S:[0,1]^{2} \rightarrow[0,1]$ is called a t-conorm if it satisfies, for all $x, y, z \in$ $[0,1]$, the following conditions

(S1) $S(x, y)=S(y, x)$,

(S2) $S(x, S(y, z))=S(S(x, y), z)$,

(S3) $S(x, y) \leq S(x, z)$ for $y \leq z$, i.e., $S(x, \cdot)$ is isotone,

(S4) $S(x, 0)=x$. 
Definition 2.4 ([4, p. 3], [11, Definition 11.3]). An antitone function $N:[0,1] \rightarrow[0,1]$ is called a fuzzy negation if $N(0)=1, N(1)=0$. Moreover, a fuzzy negation $N$ is called

(i) strict if it is strictly decreasing and continuous;

(ii) strong if it is an involution, i.e., $N(N(x))=x$ for all $x \in[0,1]$.

Definition 2.5 ([3, Definition 1.4.15]). Let $I$ be a fuzzy implication. The function $N_{I}$ defined by $N_{I}(x)=I(x, 0), x \in[0,1]$, is called a natural negation of $I$.

Definition 2.6 ([3, Definition 2.5.1]). A function $I:[0,1]^{2} \rightarrow[0,1]$ is called an R-implication if there exists a t-norm $T$ such that

$$
I(x, y)=\sup \{t \in[0,1] \mid T(x, t) \leq y\}, \quad x, y \in[0,1] .
$$

An R-implication generated by a t-norm $T$ will be denoted by $I_{T}$. The set of all R-implications we denote by $\mathbb{I}_{\mathbb{T}}$.

Definition 2.7 ([3, Definition 2.4.1]). A function $I:[0,1]^{2} \rightarrow[0,1]$ is called an (S,N)-implication if there exist a t-conorm $S$ and a negation $N$ such that

$$
I(x, y)=S(N(x), y), \quad x, y \in[0,1] .
$$

An (S,N)-implication generated by a t-conorm $S$ and fuzzy negation $N$ will be denoted by $I_{S, N}$. The set of all $(\mathrm{S}, \mathrm{N})$-implications we denote by $\mathbb{I}_{\mathbb{S}, \mathbb{N}}$.

Definition 2.8 ([12]). A copula (specifically, a 2copula) is a function $C:[0,1]^{2} \rightarrow[0,1]$ which satisfies the following conditions

(C1) $C(x, 0)=C(0, y)=0$, for all $x, y \in[0,1]$,

(C2) $C(x, 1)=x$, for all $x \in[0,1]$,

(C3) $C(1, y)=y$, for all $y \in[0,1]$,

(C4) $C\left(x_{2}, y_{2}\right)-C\left(x_{2}, y_{1}\right)-C\left(x_{1}, y_{2}\right)+C\left(x_{1}, y_{1}\right) \geq$ 0 , for all $x_{1}, x_{2}, y_{1}, y_{2} \in[0,1]$ such that $x_{1} \leq$ $x_{2}, y_{1} \leq y_{2}$.

Example 2.9. The following are some basic copulas investigated in the literature.

(a) $C(x, y)=M(x, y)=\min (x, y)$.

(b) $C(x, y)=W(x, y)=\max (x+y-1,0)$.

(c) $C(x, y)=\Pi(x, y)=x \cdot y$.

(d) the Farlie-Gumbel-Morgenstern family, $\operatorname{FGM}(\theta)$, where $\theta \in[-1,1]$ :

$$
C_{\theta}(x, y)=x \cdot y+\theta x \cdot y(1-x)(1-y) .
$$

It can be shown that every copula is bounded by the so-called Fréchet-Hoeffding bounds, i.e., for any copula $C$ and for all $x, y \in[0,1]$ the following inequalities hold

$$
W(x, y) \leq C(x, y) \leq M(x, y) .
$$

The notion of conditional copula was applied for defining probabilistic implications and probabilistic S-implications.

Definition 2.10 (see $[7,10]$ ). Let $C$ be a copula. A function $I_{C}:[0,1]^{2} \rightarrow[0,1]$ given by

$$
I_{C}(x, y)=\left\{\begin{array}{lll}
1 & \text { if } & x=0 \\
\frac{C(x, y)}{x} & \text { if } & x>0
\end{array}\right.
$$

is called a probabilistic implication (based on a copula $C$ ).

Definition 2.11 (see $[7,10]$ ). Let $C$ be a copula. A function $\tilde{I}_{C}:[0,1]^{2} \rightarrow[0,1]$ given by

$$
\tilde{I}_{C}(x, y)=C(x, y)-x+1,
$$

is called a probabilistic S-implication (based on a copula $C$ ).

These two families of multi-valued implications have been deeply investigated in [2].

Definition 2.12 ([12]). Let $C$ be a copula. A function $C^{*}:[0,1]^{2} \rightarrow[0,1]$ defined by

$$
C^{*}(x, y)=x+y-1+C(1-x, 1-y)
$$

is called the survival copula (based on a copula $C$ ).

The notion of survival copula was applied for defining survival implications and survival Simplications.

Definition 2.13 ([9]). Let $C$ be a copula. A function $I_{C}^{*}:[0,1]^{2} \rightarrow[0,1]$ given by

$$
\begin{aligned}
I_{C}^{*}(x, y) & = \begin{cases}1, & x=0 \\
\frac{C^{*}(x, y)}{x}, & x>0\end{cases} \\
& = \begin{cases}1, & x=0, \\
\frac{x+y-1+C(1-x, 1-y)}{x}, & x>0,\end{cases}
\end{aligned}
$$

for all $x, y \in[0,1]$, is called a survival implication (based on a copula $C$ ).

The set of all survival implications will be denoted by $\mathbb{I}_{\mathbb{C}}^{*}$.

Definition 2.14 ([9]). Let $C$ be a copula. A function $\tilde{I}_{C}^{*}:[0,1]^{2} \rightarrow[0,1]$ given by

$$
\tilde{I}_{C}^{*}(x, y)=C^{*}(x, y)-x+1=C(1-x, 1-y)+y,
$$

for all $x, y \in[0,1]$, is called a survival $\mathbf{S}$ implication (based on a copula $C$ ).

The set of all survival S-implications will be denoted by $\tilde{\mathbb{I}}_{\mathbb{C}}^{*}$.

The following result is obvious.

Remark 2.15 ([9, Lemma 2]). If $C^{*}=C$, then the survival implication based on $C$ is equal to the probabilistic implication based on the same copula, and the survival S-implication based on $C$ is equal to the probabilistic S-implication based on the same copula, i.e., $I_{C}^{*}=I_{C}$ and $\tilde{I}_{C}^{*}=\tilde{I}_{C}$. 
Example 2.16. Let $C(x, y)=\min (\sqrt{x} y, x \sqrt{y})$, then function $C$ is a copula that belongs to the Cuadras-Augé family (see [12]). Furthermore, $C^{*} \neq$ $C$. Indeed, for $x=y=\frac{1}{3}$ we obtain that $C^{*} \neq C$. Hence, $\tilde{I}_{C}^{*} \neq \tilde{I}_{C}$ and $I_{C}^{*} \neq I_{C}$.

It is worth noting that a survival implication is not necessarily a fuzzy implication. To guarantee that a survival implication is also a fuzzy implication we need to add condition (I1) that $I_{C}^{*}$ is antitone with respect to the first variable (other conditions in Definition 2.1 are satisfied by any survival implication, see [9]).

In reference to Example 2.9 we can easily conclude that applying copulas $M$ or $\Pi$ (which are equal to their survival versions) we obtain well known fuzzy implications. Actually,

$$
I_{M}^{*}(x, y)=I_{\mathbf{G G}}(x, y)= \begin{cases}1, & x \leq y \\ \frac{y}{x}, & x>y\end{cases}
$$

i.e., $I_{M}^{*}=I_{M}=I_{\mathrm{GG}}$ is the Goguen implication, while

$$
I_{\Pi}^{*}(x, y)= \begin{cases}1, & x=0 \\ y, & x>0\end{cases}
$$

i.e., $I_{\Pi}^{*}=I_{\Pi}=I_{\mathbf{D}}$ is the least $(\mathrm{S}, \mathrm{N})$-implication (see [3]). However, the survival implication

$$
I_{W}^{*}(x, y)=I_{W}(x, y)= \begin{cases}1, & x=0 \\ \frac{\max (x+y-1,0)}{x}, & x>0\end{cases}
$$

based on the lower Fréchet-Hoeffding bound $W$ is not a fuzzy implication. On the other hand survival implications based on copulas belonging to the Farlie-Gumbel-Morgenstern family $\operatorname{FGM}(\theta)$ are fuzzy implications only for $\theta \geq 0$. It should be stressed that any survival S-implication - contrary to survival implication - is a fuzzy implication. To finish this preliminary section we examine the relation between two new families of implications.

Proposition 2.17. The family of all survival implications and the family of all survival $S$ implications are disjoint, i.e.,

$$
\mathbb{I}_{\mathbb{C}}^{*} \cap \tilde{\mathbb{I}}_{\mathbb{C}}^{*}=\emptyset .
$$

Proof. Suppose that there exist two copulas $C_{1}$ and $C_{2}$ such that $I_{C_{1}}^{*}=\tilde{I}_{C_{2}}^{*} \in \mathbb{I}_{\mathbb{C}}^{*} \cap \tilde{\mathbb{I}}_{\mathbb{C}}^{*}$. Therefore, we have for all $x, y \in[0,1], x \neq 0$

$$
\frac{x+y-1+C_{1}(1-x, 1-y)}{x}=C_{2}(1-x, 1-y)+y .
$$

Substituting $y=0$ in the above equation we obtain that $0=\frac{0}{x}=1-x$, which is not true for $x \in(0,1)$. This contradicts our assumption and finishes the proof.

\section{The Laws of Contraposition}

\subsection{Introduction}

One of the most important tautologies in the classical two-valued logic is the law of contraposition:

$$
p \rightarrow q \equiv \neg q \rightarrow \neg p .
$$

Since the classical negation satisfies the law of double negation $(\neg(\neg p) \equiv p)$, the following laws are also tautologies in the classical logic:

$$
\begin{aligned}
\neg p \rightarrow q & \equiv \neg q \rightarrow p, \\
p \rightarrow \neg q & \equiv q \rightarrow \neg p .
\end{aligned}
$$

Natural generalizations of those classical tautologies to fuzzy logic which play an important role in various applications are based on fuzzy negations and fuzzy implications.

Definition 3.1 ([3, Definition 1.5.1]). Let $I$ denote a fuzzy implication and $N$ be a fuzzy negation. We say that $I$ satisfies

(i) the law of contraposition (or contrapositive symmetry) with respect to $N$ if

$$
I(x, y)=I(N(y), N(x)), \quad x, y \in[0,1] ;
$$

(ii) the law of left contraposition with respect to $N$ if

$$
I(N(x), y)=I(N(y), x), \quad x, y \in[0,1]
$$

(iii) the law of right contraposition with respect to $N$ if

$$
I(x, N(y))=I(y, N(x)), \quad x, y \in[0,1] .
$$

If $I$ satisfies the contrapositive symmetry (left, right) with respect to $N$, then we also denote it by $\mathrm{CP}(N)(\mathrm{L}-\mathrm{CP}(N)$, R-CP $(N)$, respectively).

It can be proved that the three properties introduced in Definition 3.1 are equivalent when $N$ is a strong negation (see [3, Proposition 1.5.3]).

\subsection{The Laws of Contraposition for Survival Implications}

Let us firstly consider the natural negation based on a survival implication. The following result may be proved.

Lemma 3.2. Let $I_{C}^{*}$ be a survival implication based on a copula $C$. The natural negation $N_{I_{C}^{*}}$ based on $I_{C}^{*}$ is the least fuzzy negation (cf. [3, p. 14]), i.e.,

$$
N_{I_{C}^{*}}(x)=N_{\mathbf{D} \mathbf{1}}(x)= \begin{cases}1, & x=0, \\ 0, & x>0,\end{cases}
$$

for all $x \in[0,1]$. 
Proof. For any survival implication $I_{C}^{*}$ and any $x \in$ $[0,1]$ we have

$$
\begin{aligned}
N_{I_{C}^{*}}(x) & =I_{C}^{*}(x, 0)= \begin{cases}1, & x=0 \\
\frac{x-1+C(1-x, 1)}{x}, & x>0\end{cases} \\
& =N_{\mathbf{D} \mathbf{1}}(x) .
\end{aligned}
$$

Now we are able to investigate the laws of contraposition for survival implications.

Theorem 3.3. No survival implication satisfies the law of contraposition (CP) or the law of left contraposition (L-CP) with any negation $N$.

Proof. Let us firstly recall that a function $I:[0,1]^{2} \rightarrow[0,1]$ satisfies the left neutrality property (see [3, Definition 1.3.1]) if

$$
I(1, y)=y \quad y \in[0,1] .
$$

Observe that

$$
I_{C}^{*}(1, y)=\frac{1+y-1+C(1-1,1-y)}{1}=y,
$$

so each survival implication $I_{C}^{*}$ satisfies the left neutrality property (NP). However, by Lemma 3.2 the only natural negation $N_{I_{C}^{*}}$ based on a survival implication is $N_{\mathbf{D} 1}$ which is not a strong negation. It is well-known (see [3, Corollary 1.5.5]) that if the natural negation based $N_{I}$ on a function $I$ satisfying (NP) is not strong, then $I$ does not satisfy (CP) with any fuzzy negation. Consequently, $I_{C}^{*}$ does not satisfy (CP) with any fuzzy negation.

It could be also shown that if $N_{I}$ based on a function $I$ satisfying (NP) is not a continuous negation, then $I$ does not satisfy (L-CP) with any fuzzy negation [3, Corollary 1.5.15]. Since $N_{I_{C}^{*}}=N_{\mathbf{D} 1}$ is not a continuous negation we may conclude that $I_{C}^{*}$ does not satisfy (L-CP) with any fuzzy negation.

Theorem 3.4. Every survival implication satisfies (R-CP) only with respect to the least fuzzy negation $N_{D 1}$.

Proof. One can easily check that any survival implication satisfies (R-CP) with $N_{\mathbf{D} \mathbf{1}}$. Indeed,

$$
\begin{aligned}
I_{C}^{*} & \left(x, N_{\mathbf{D} \mathbf{1}}(y)\right)= \\
& = \begin{cases}1, & x=0 \\
\frac{x+N_{D 1}(y)-1+C\left(1-x, 1-N_{D 1}(y)\right)}{x}, & x>0\end{cases} \\
& = \begin{cases}1, & x=0 \\
\frac{x+C(1-x, 0)}{x}, & x>0 \text { and } y=0 \\
\frac{x-1+C(1-x, 1)}{x}, & x>0 \text { and } y>0\end{cases} \\
& = \begin{cases}1, & x=0 \\
1, & x>0 \text { and } y=0 \\
0, & x>0 \text { and } y>0\end{cases} \\
& =I_{C}^{*}\left(y, N_{\mathbf{D} \mathbf{1}}(x)\right) .
\end{aligned}
$$

Thus, actually, any survival implication $I_{C}^{*}$ satisfies (R-CP) with $N_{\mathbf{D} \mathbf{1}}$. As it was mentioned above, $I_{C}^{*}$ satisfies the left neutrality property (see [9, Lemma 9]), but it may be showed (see [3, Lemma 1.5.21]) that if a function $I:[0,1]^{2} \rightarrow[0,1]$ satisfies (NP) and (R-CP) with a fuzzy negation $N$, then $I$ satisfies (I3) and $N=N_{I}$. Therefore, in our case we obtain that for any survival implication $I_{C}^{*}$ there exists a unique natural negation $N_{I_{C}^{*}}=N_{\mathbf{D} \mathbf{1}}$ such that the law of right contraposition holds.

\subsection{The Laws of Contraposition for Survival S-Implications}

Similarly as before, we start our considerations in this subsection by examining the natural negation based on a survival S-implication.

Lemma 3.5. Let $\tilde{I}_{C}^{*}$ be a survival $S$-implication based on a copula $C$. The natural negation $N_{\tilde{I}_{C}^{*}}$ is the strong classical negation, i.e., for all $x \in[0,1]$ we have

$$
N_{\tilde{I}_{C}^{*}}(x)=N_{\mathbf{C}}(x)=1-x .
$$

Proof. For any survival S-implication $\tilde{I}_{C}^{*}$ and all $x \in$ $[0,1]$ we have

$$
\begin{aligned}
N_{\tilde{I}_{C}^{*}}(x) & =\tilde{I}_{C}^{*}(x, 0)=C(1-x, 1)+0=1-x \\
& =N_{\mathbf{C}}(x) .
\end{aligned}
$$

Proposition 3.6. Let $\tilde{I}_{C}^{*}$ be a survival $S$ implication. If $\tilde{I}_{C}^{*}$ satisfies the $(\mathrm{CP})$ with respect to a fuzzy negation $N$, then $N$ is the strong classical negation $N_{\mathbf{C}}$.

Proof. It is easy to show that any survival S-implication satisfies the left neutrality property (NP). Thus, knowing that if a function $I:[0,1]^{2} \rightarrow[0,1]$ satisfies $(\mathrm{NP})$ and $(\mathrm{CP})$ with respect to a fuzzy negation $N$, then $N=N_{I}$ is a strong negation (see [3, Lemma 1.5.4 (v)]). Hence, we conclude that $N=N_{\tilde{I}_{C}^{*}}=N_{\mathbf{C}}$.

Combining (2) and (CP) we obtain immediately

Corollary 3.7. The law of contraposition (CP) (with respect to $N_{\mathbf{C}}$ ) holds for a survival $S$ implication $\tilde{I}_{C}^{*}$ based on a copula $C$ if and only if $C$ satisfies the following equation

$$
C(x, y)-x+1=C(1-y, 1-x)+y,
$$

for all $x, y \in[0,1]$.

Please observe that the above functional equation is strictly connected with the following equation

$$
x+y-C(x, y)=1-C(1-x, 1-y),
$$

which often occurs in the literature connected with copulas (cf [12, Theorem 2.7.3] or [6, p. 97]) and for which we know some partial solutions, in particular 
when $C$ is an Archimedean continuous t-norm [5]. Solving (6) we may obtain a characterization of a family of copulas leading to survival S-implications for which the law of contraposition (with respect to $N_{\mathbf{C}}$ ) holds. However, finding that (general) solution is still an open problem. At this moment we can list several examples of such copulas $C$ that the survival S-implications $\tilde{I}_{C}^{*}$ satisfies $(\mathrm{CP})$, e.g. survival Simplications based on copulas $\Pi, M, W$ or copulas from the family $\operatorname{FGM}(\theta)$.

Proposition 3.8. Let $\tilde{I}_{C}^{*}$ be a survival $S$ implication. If $\tilde{I}_{C}^{*}$ satisfies the (L-CP) or (R-CP) with respect to a fuzzy negation $N$, then $N$ is the strong classical negation $N_{\mathbf{C}}$.

Proof. Since any survival S-implication satisfies the left neutrality property, then, assuming (L-CP), in view of $\left[3\right.$, Lemma 1.5.14 (ii)], we have $N_{\tilde{I}_{C}^{*}}(N(x))=$ $x$ for each $x \in[0,1]$. It means that $N_{\tilde{I}_{C}^{*}}=N_{\mathbf{C}}$ and consequently $N=N_{C}$. Similarly, assuming (R-CP), by [3, Lemma 1.5.21], we conclude that $N=N_{\tilde{I}_{C}^{*}}=N_{\mathbf{C}}$.

\section{T-Conditionality}

An implication operator plays an important role in the deductive process of a logic which is usually realized by some rules of inference. Modus ponens is one such rule of inference, wherein given two classical logic propositions $A \rightarrow B$ and $A$ we infer $B$. A similar rule of inference in the case of dealing with fuzzy propositions is called the generalized modus ponens (GMP) wherein given two fuzzy propositions $A \rightarrow B$ and $A^{\prime}$ we infer $B^{\prime}$. The highlight of this inference is even if $A^{\prime} \neq A$ we still will be able to infer a reasonable conclusion $B^{\prime}$. One of the conditions that any inference scheme employed to realize GMP is expected to satisfy is that the GMP should coincide with $T$-conditionality (see [13]) in the case $A^{\prime}=A$, i.e., $B$ should be $B^{\prime}$.

Definition 4.1. An implication $I$ and a t-norm $T$ satisfy $T$-conditionality if and only if

$$
T(x, I(x, y)) \leq y, \quad \text { for all } x, y \in[0,1] .
$$

The following theorem characterizes shortly survival implications which satisfy (TC) with all tnorms.

Theorem 4.2. If $I_{C}^{*}$ is a survival implication based on a copula $C$, then the following statements are equivalent:

(i) $I_{C}^{*}$ satisfies (TC) with any t-norm $T$.

(ii) $C(1-u, 1-v) \leq(1-u)(1-v)$ for all $u, v \in[0,1]$ such that $u>v$.

Proof. (i) $\Longrightarrow$ (ii): If $I_{C}^{*}$ satisfies (TC) with any tnorm, then in particular it satisfies (TC) with minimum

$$
\begin{aligned}
& \min \left(u, I_{C}^{*}(u, v)\right)= \\
& \quad=\min \left(u, \frac{u+v-1+C(1-u, 1-v)}{u}\right) \\
& \quad \leq v .
\end{aligned}
$$

If $\min \left(u, \frac{u+v-1+C(1-u, 1-v)}{u}\right)=u$, then $u \leq v$, which is a contradiction with the assumption $u>v$. Thus, $\min \left(u, \frac{u+v-1+C(1-u, 1-v)}{u}\right)=$ $\frac{u+v-1+C(1-u, 1-v)}{u} \leq v$. Therefore, we have $C(1-u, 1-v) \leq(1-u)(1-v)$ for $u>v$.

(ii) $\Longrightarrow(\mathrm{i})$ : Since the greatest t-norm is the minimum we have for each t-norm $T$

$$
T\left(u, I_{C}^{*}(u, v)\right) \leq \min \left(u, I_{C}^{*}(u, v)\right) \leq u .
$$

Thus if $u \leq v$, then obviously inequality (TC) is satisfied for all implications and t-norms. Therefore, only situation when $u>v$ will be of our interest. Every t-norm $T$ is increasing with respect to both variables. Thus, for $C(1-u, 1-v) \leq(1-u)(1-v)$ we have

$$
\begin{aligned}
& T\left(u, I_{C}^{*}(u, v)\right) \\
& \quad=T\left(u, \frac{u+v-1+C(1-u, 1-v)}{u}\right) \\
& \quad \leq \min \left(u, \frac{u+v-1+(1-u)(1-v)}{u}\right) \\
& \quad=v,
\end{aligned}
$$

which completes the proof.

Using Theorem 4.2 we can easily check which survival implications satisfy (TC) with all t-norms. For instance, functions based on copulas $\Pi, W$ and functions based on copulas from families $\operatorname{FGM}(\theta)$, for $\theta \in[-1,0]$, satisfy (TC) for any t-norm. However, functions based on copula $M$ or based on copulas from families $\operatorname{FGM}(\theta)$ for $\theta \in(0,1]$, do not satisfy (TC).

Contrary to survival implications none of survival S-implications satisfies (TC) for all t-norms. However, each survival S-implication satisfies (TC) for at least one t-norm.

Proposition 4.3. No probabilistic S-implication satisfies (TC) for the t-norm $T_{M}$.

Proof. Suppose that function $\tilde{I}_{C}^{*}$ satisfies (TC) for t-norm $T_{M}$. Then for $u>v$,

$$
\min (u, v+C(1-u, 1-v)) \leq v,
$$

so, $v+C(1-u, 1-v) \leq v$. Thus, $C(1-u, 1-v)=$ 0 for $u>v$. Using the lower Fréchet-Hoeffding's bound for $u=0.8$ and $v=0.1$, we obtain that

$0.1=\max (1-0.8-0.1,0) \leq C(1-0.8,1-0.1)=0$.

This is a contradiction with our assumption. 
Proposition 4.4. Each probabilistic S-implication satisfies (TC) for the t-norm $W$.

Proof. Suppose that function $\tilde{I}_{C}^{*}$ does not satisfy (TC) for t-norm $W$. Then,

$$
\max (u+v+C(1-u, 1-v)-1,0)>v,
$$

for some $u, v \in[0,1]$. Hence, $C(1-u, 1-v)>1-u$ this is contradiction with upper Fréchet-Hoeffding's bound.

\section{Intersections Between Families of Implications}

\subsection{Intersections with R-Implications}

Lemma 5.1. If a copula $C$ is idempotent, then $C=$ $M$.

Proof. Let copula $C$ will be idempotent. By upper Fréchet-Hoeffding bound $C(x, y) \leq \min (x, y)$ for all $x, y \in[0,1]$. Suppose that $C\left(x_{0}, y_{0}\right)<\min \left(x_{0}, y_{0}\right)$ for some $x_{0}, y_{0} \in(0,1)$ such that $x_{0}<y_{0}$. Using (C4) we obtain $x_{0}>C\left(x_{0}, y_{0}\right) \geq C\left(x_{0}, x_{0}\right)=x_{0}$ and this is contradiction with our assumption. In the case when $y_{0}<x_{0}$ the proof is analogue.

Theorem 5.2. The only survival implication which is an R-implication is the Goguen implication (3), i.e.,

$$
\mathbb{I}_{\mathbb{T}} \cap \mathbb{I}_{\mathbb{C}}^{*}=\left\{I_{\mathrm{GG}}\right\} .
$$

Proof. If $I_{C}^{*}(x, y)$ is an R-implication, then $I_{C}^{*}$ satisfies the identity principle, i.e., $I_{C}^{*}(x, x)=1$ (see [3, Theorem 2.5.4]). This implies that

$$
\begin{aligned}
& \frac{x+x-1+C(1-x, 1-x)}{x}=1 \\
& \Longleftrightarrow 2 x-1+C(1-x, 1-x)=x \\
& \Longleftrightarrow C(1-x, 1-x)=1-x
\end{aligned}
$$

for all $x>0$. Putting $y:=1-x$ we have $C(y, y)=y$ for all $y<1$. But $C(1,1)=1$, so $C(y, y)=y$ for all $y \in[0,1]$, i.e., $C$ is idempotent. However, the only such copula is $M$, and we already know that $I_{M}^{*}=I_{\mathrm{GG}}$. The Goguen implication is an Rimplication based on the t-norm $\Pi$ (see [3, p. 70]). Thus we obtain that $\mathbb{I}_{\mathbb{T}} \cap \mathbb{I}_{\mathbb{C}}^{*}=\left\{I_{\mathrm{GG}}\right\}$.

Theorem 5.3. The only survival S-implication which is an R-implication is the Lukasiewicz implication, i.e.,

$$
\mathbb{I}_{\mathbb{T}} \cap \tilde{\mathbb{I}}_{\mathbb{C}}^{*}=\left\{I_{\mathbf{L K}}\right\} .
$$

Proof. If $\tilde{I}_{C}^{*}(x, y)$ is an R-implication, then $\tilde{I}_{C}^{*}$ satisfies the identity principle, i.e., $\tilde{I}_{C}^{*}(x, x)=1$ (see [3, Theorem 2.5.4]). This implies that $C(1-x, 1-x)+$ $x=1$, so putting $y:=1-x$ we have $C(y, y)=y$ for all $y \in[0,1]$, i.e., $C$ is idempotent. However, the only idempotent copula is $M$, but then $\tilde{I}_{M}^{*}=I_{\mathbf{L K}}$. Of course the Eukasiewicz implication is an R-implication generated from the t-norm $W$ (See $\left[3\right.$, p. 70]). Thus, $\mathbb{I}_{\mathbb{T}} \cap \tilde{\mathbb{I}}_{\mathbb{C}}^{*}=\left\{I_{\mathbf{L K}}\right\}$.
The results related to intersections between Rimplications and survival implications or survival S-implications, respectively, are represented by diagrams in Figure 1.

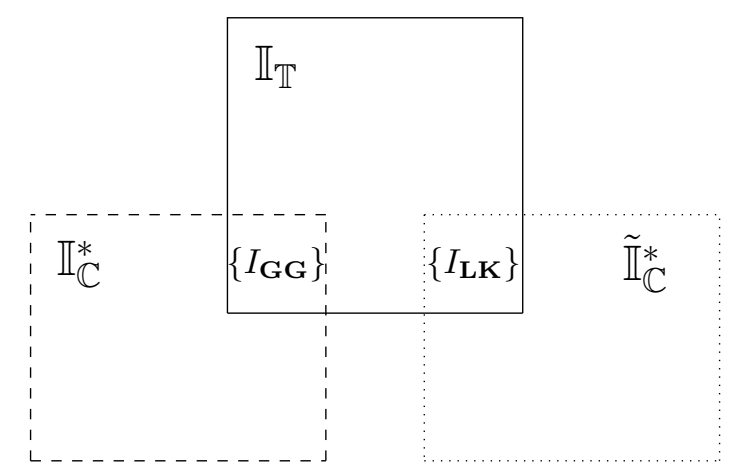

Figure 1: Intersections between $\mathbb{I}_{\mathbb{T}}, \mathbb{I}_{\mathbb{C}}^{*}$ and $\tilde{\mathbb{I}}_{\mathbb{C}}^{*}$

\subsection{Intersections with $(\mathrm{S}, \mathrm{N})$-Implications}

Theorem 5.4. The only survival implication which is an $(S, N)$-implication is the least $(S, N)$ implication $I_{\mathbf{D}}$, i.e.,

$$
\mathbb{I}_{\mathbb{S}, \mathbb{N}} \cap \mathbb{I}_{\mathbb{C}}^{*}=\left\{I_{\mathbf{D}}\right\}
$$

Proof. If a survival implication $I_{C}^{*}$ based on a copula $C$ is also an $(\mathrm{S}, \mathrm{N})$-implication, i.e., $I_{C}^{*} \in \mathbb{I}_{\mathbb{S}, \mathbb{N}} \cap \mathbb{I}_{\mathbb{C}}^{*}$, then there exist a t-conorm $S$ and negation $N$ such that $I_{C}^{*}(x, y)=S(N(x), y)$ for all $x, y \in[0,1]$. Therefore, $I_{C}^{*}(x, 0)=S(N(x), 0)=N(x)$, so by Lemma $3.2 N(x)=N_{\mathbf{D 1}}(x)$. This implies that $I_{C}^{*}(x, y)=S(0, y)=y$ for $x>0$. Thus, by (1), for $x>0$ we have $C(1-x, 1-y)=1-x-y+x y=$ $(1-x)(1-y)$, so one can easily deduce that $C=\Pi$. Hence, $I_{C}^{*}=I_{\Pi}^{*}=I_{\mathbf{D}}$ is the least $(\mathrm{S}, \mathrm{N})$-implication, which proves the theorem.

Since survival S-implications and (S,N)implications are derived from the same classical tautology

$$
A \rightarrow B \equiv \neg A \wedge B,
$$

one may expect that both families of implications are somehow related. Suppose $I \in \mathbb{I}_{\mathbb{S}, \mathbb{N}} \cap \tilde{\mathbb{I}}_{\mathbb{C}}^{*}$. Then, by (2), there exists a copula $C$, a t-conorm $S$ and negation $N$ such that

$$
C(1-x, 1-y)+y=S(N(x), y) .
$$

Substituting $y=0$ into (7) we obtain that

$$
1-x=S(N(x), 0)=N(x),
$$

which means that $N(x)=1-x=N_{\mathbf{C}}(x)$. Therefore, equation (7) may be expressed, for all $x, y \in$ $[0,1]$, as

$$
C(1-x, 1-y)+y=S(1-x, y) .
$$

Rewriting (8) we have

$$
C(1-x, 1-y)=S(1-x, y)-y .
$$


It is easy to check, that so defined function satisfies conditions (C1)-(C3) in Definition 2.8 for any t-conorm $S$. However, to ensure that $(\mathbf{C 4})$ in Definition 2.8 is also satisfied we obtain the following requirement for $S$ for all $x_{1}, x_{2}, y_{1}, y_{2} \in[0,1]$, such that $x_{1} \leq x_{2}, y_{1} \leq y_{2}$ :

$$
\begin{aligned}
& C\left(1-x_{2}, 1-y_{2}\right)-C\left(1-x_{2}, 1-y_{1}\right) \\
& -C\left(1-x_{1}, 1-y_{2}\right)+C\left(1-x_{1}, 1-y_{1}\right) \geq 0 \\
& \Longleftrightarrow \\
& S\left(1-x_{2}, y_{2}\right)-y_{2}-S\left(1-x_{2}, y_{1}\right)+y_{1} \\
& -S\left(1-x_{1}, y_{2}\right)+y_{2}+S\left(1-x_{1}, y_{1}\right)-y_{1} \geq 0 \\
& \Longleftrightarrow \\
& S\left(x_{1}^{\prime}, y_{2}\right)-S\left(x_{1}^{\prime}, y_{1}\right)-S\left(x_{2}^{\prime}, y_{2}\right)+S\left(x_{2}^{\prime}, y_{1}\right) \geq 0,
\end{aligned}
$$

where $x_{1}^{\prime}=1-x_{2}$ and $x_{2}^{\prime}=1-x_{1}$. Since $x_{1} \leq$ $x_{2}$ thus the last inequality holds for any $x_{1}^{\prime} \leq x_{2}^{\prime}$. To sum up these results we have actually proved a lemma given below.

Lemma 5.5. An (S,N)-implication $I_{S, N}$ is a survival $S$-implication if and only if $N=N_{C}$ and the t-conorm $S$ satisfies the following condition

$$
S\left(x_{1}, y_{1}\right)+S\left(x_{2}, y_{2}\right)-S\left(x_{1}, y_{2}\right)-S\left(x_{2}, y_{1}\right) \leq 0
$$

for all $x_{1}, x_{2}, y_{1}, y_{2} \in[0,1]$ such that $x_{1} \leq x_{2}, y_{1} \leq$ $y_{2}$.

This result leads to the next theorem.

Theorem 5.6. An (S,N)-implication $I_{S, N}$ is a survival $S$-implication if and only if $N=N_{C}$ and the t-conorm $S$ is dual to the t-norm $T$ which is a copula.

Proof. Let $T$ be a t-norm such that $S(x, y)=1-$ $T(1-x, 1-y)$. Then for $x_{1}, x_{2}, y_{1}, y_{2} \in[0,1]$, such that $x_{1} \leq x_{2}$ and $y_{1} \leq y_{2}$ we have

$$
\begin{aligned}
& S\left(x_{1}, y_{1}\right)+S\left(x_{2}, y_{2}\right)-S\left(x_{1}, y_{2}\right)-S\left(x_{2}, y_{1}\right) \leq 0 \\
& \Longleftrightarrow \\
& -T\left(1-x_{1}, 1-y_{1}\right)-T\left(1-x_{2}, 1-y_{2}\right) \\
& +T\left(1-x_{1}, 1-y_{2}\right)+T\left(1-x_{2}, 1-y_{1}\right) \leq 0 \\
& \Longleftrightarrow \\
& T\left(x_{1}^{\prime}, y_{1}^{\prime}\right)+T\left(x_{2}^{\prime}, y_{2}^{\prime}\right)-T\left(x_{1}^{\prime}, y_{2}^{\prime}\right)-T\left(x_{2}^{\prime}, y_{1}^{\prime}\right) \geq 0,
\end{aligned}
$$

where $x_{1}^{\prime}=1-x_{1}, x_{2}^{\prime}=1-x_{2}, y_{1}^{\prime}=1-y_{1}$ and $y_{2}^{\prime}=1-y_{2}$, i.e., $x_{2}^{\prime} \leq x_{1}^{\prime}$ and $y_{2}^{\prime} \leq y_{1}^{\prime}$. The last inequality is equivalent to (C4) in Definition 2.8. Other conditions, i.e., (C1)-(C3) in Definition 2.8, are implied directly from Definition 2.2. Therefore, $T$ is a copula, which proves the theorem.

Going back to (8) we may consider a reverse problem and ask, for which copula $C$ a function $S(1-x, y)=C(1-x, 1-y)+y$ is a t-conorm and hence when a survival S-implication is also an $(\mathrm{S}, \mathrm{N})$-implication. The answer is delivered by the following result.
Proposition 5.7. A survival S-implication $\tilde{I}_{C}$ based on a copula $C$ is an $(S, N)$-implication if and only if $C$ satisfies the two following equations:

$$
\begin{array}{r}
C(x, 1-y)+y=C(y, 1-x)+x, \\
C(x, 1-C(y, 1-z)-y)+C(y, 1-z) \\
=C(C(x, 1-y)+y, 1-z),
\end{array}
$$

for all $x, y, z \in[0,1]$.

Proof. Using argumentation analogous to that preceding Lemma 5.5 we have to identify the requirements on a copula $C$ which guarantee that the function $S(1-x, y)=C(1-x, 1-y)+y$ is a t-conorm. Substituting $1-x$ by $x$ we may consider a function $S(x, y)=C(x, 1-y)+y$. By Definition 2.3 we should verify conditions $(\mathbf{S 1})-(\mathbf{S} 4)$.

1. Commutativity:

$$
\begin{aligned}
S(x, y) & =S(y, x) \Longleftrightarrow \\
& C(x, 1-y)+y=C(y, 1-x)+x,
\end{aligned}
$$

which gives requirement (9).

2. Associativity:

$$
\begin{aligned}
& \quad S(x, S(y, z))=S(S(x, y), z) \\
& \Longleftrightarrow C(x, 1-C(y, 1-z)-y)+C(y, 1-z)+z \\
& =C(C(x, 1-y)+y, 1-z)+z,
\end{aligned}
$$

which gives requirement (10).

3. $S(x, \cdot)$ is isotone - but it holds since any copula $C$ is an isotone function for both arguments.

4. Zero is a neutral element of $S$ - but it holds since $S(x, 0)=C(x, 1)+0=x$.

By Corollary 3.7 and condition (9) in the last lemma we obtain straightforwardly an interesting conclusion.

Corollary 5.8. Survival S-implications which are $(S, N)$-implications satisfy the law of contraposition (CP) with respect to $N_{C}$.

To sum up the results related to intersections between (S,N)-implications and survival implications or survival S-implications, respectively, they are represented by diagrams in Figure 5.2. By $\mathbb{I}_{S^{*}, N_{C}}$ we denote a set of (S,N)-implications such that $N=N_{C}$ and $S^{*}$ is t-conorm dual to t-norm being a copula (such as in Theorem 5.6). Please note, that some well known fuzzy implications, like $I_{\mathbf{L K}}$, the Reichenbach implication $I_{\mathbf{R C}}(x, y)=1-x+x y$ or the Kleene-Dienes implication $I_{K D}(x, y)=\max (1-$ $x, y$ ) belong to $\mathbb{I}_{S^{*}, N_{C}}$ (see $\left.[3]\right)$. 


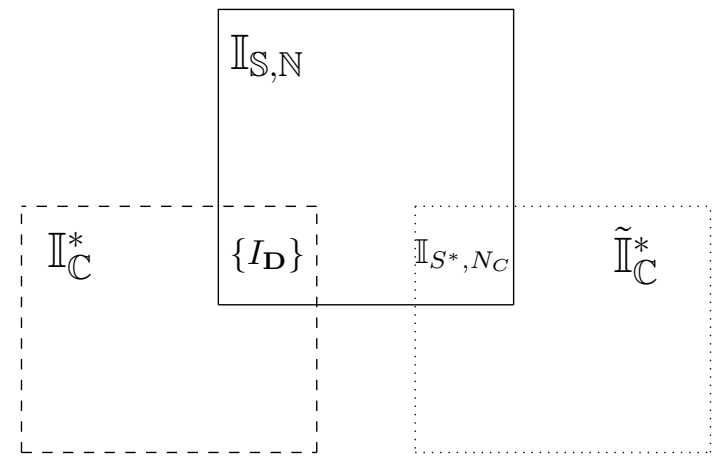

Figure 2: Intersections between $\mathbb{I}_{\mathbb{S}, \mathbb{N}}, \mathbb{I}_{\mathbb{C}}^{*}$ and $\tilde{\mathbb{I}}_{\mathbb{C}}^{*}$

\section{Conclusion}

In this paper we have examined some interesting properties of survival implications and survival S-implications, like laws of contrapositions (CP), (L-CP), (R-CP), T-conditionality (TC) and intersections between survival implications or survival S-implications and R-implications and (S,N)-implications.

However, some questions are still open. For instance, is there any particular family of copulas which satisfies conditions (9) and (10)? Is there any particular family of copulas which satisfies condition (6)?

\section{References}

[1] M. Baczyński, G. Beliakov, H. Bustince and A. Pradera, editors, Advances in Fuzzy Implication Functions, Studies in Fuzziness and Soft Computing, Vol. 300, Springer, Berlin Heidelberg, 2013.

[2] M. Baczyński, P. Grzegorzewski and W. Niemyska, Laws of contraposition and law of importation for probabilistic implications and probabilistic S-implications, In A. Laurent, O. Strauss, B. Bouchon-Meunier and R.R. Yager, editors, Information Processing and Management of Uncertainty in KnowledgeBased Systems, 15th International Conference, IPMU 2014, Montpellier, France, July 15-19, 2014, Proceedings, Part I, Communications in Computer and Information Science 442, pages 158-167, Springer International Publishing, 2014.

[3] M. Baczyński and B. Jayaram, Fuzzy Implications, Studies in Fuzziness and Soft Computing, Vol. 231, Springer, Berlin Heidelberg, 2008.

[4] J. Fodor and M. Roubens, Fuzzy Preference Modelling and Multicriteria Decision Support, Kluwer, Dordrecht, 1994.
[5] M.J. Frank, On the simultaneous associativity of $F(x, y)$ and $x+y-F(x, y)$, Aequationes Math., 19:194-226, 1979.

[6] M. Grabisch, J.-L. Marichal, R. Mesiar and E. Pap, Aggregation Functions, Encyclopedia of Mathematics and Its Applications, Vol. 127, Cambridge University Press, Cambridge, 2009.

[7] P. Grzegorzewski, Probabilistic implications, In S. Galichet, J. Montero and G. Mauris, editors, Proc. of the rth Conference on European Society for Fuzzy Logic and Technology (EUSFLAT-2011) and LFA-2011 Aix-lesBains, France, July 18-22, 2011, Advances in Intelligent Systems Research, Vol. 1, pages 254-258, Atlantis Press, 2011.

[8] P. Grzegorzewski, On the properties of probabilistic implications, In P. Melo-Pinto, P. Couto, C. Serôdio, J. Fodor and B. De Baets, editors, Eurofuse 2011, Workshop on Fuzzy Methods for Knowledge-Based Systems, Advances in Intelligent and Soft Computing, Vol. 107, pages 67-78, Springer-Verlaq, 2011.

[9] P. Grzegorzewski, Survival Implications, In S. Greco, B. Bouchon-Meunier, G. Coletti, M. Fedrizzi, B. Matarazzo and R.R. Yager, editors, Advances in Computational Intelligence, Part II (Proc. 14th International Conference, IPMU 2012, Catania, Italy, July 9-13, 2012), Communications in Computer and Information Science 298, pages 335-344, Springer-Verlag, 2012.

[10] P. Grzegorzewski, Probabilistic implications, Fuzzy Sets and Systems, 226:53-66, 2013.

[11] E.P. Klement, R. Mesiar and E. Pap, Triangular norms, Kluwer, Dordrecht, 2000.

[12] R.B. Nelsen, An Introduction to Copulas, 2nd Edition, Springer, New York, 2006.

[13] L.A. Zadeh, Outline of a new approach to the analysis of complex systems and decision processes, IEEE Trans. Syst. Man Cybernet., 9:28-44, 1973. 